\title{
Some problems in differential geometry and topology
}

\author{
S.K. Donaldson
}

June 5, 2008

This does not attempt to be a systematic overview, or a to present a comprehensive list of problems. We outline some questions in three different areas which seem to the author interesting. Experts will learn little new; our goal is to give some picture of the fields for non-specialists.

\section{Low-dimensional topology and symplectic topol- ogy}

The general setting here is that one would like to understand the classification of manifolds, and related objects such as knots in 3-space. The "classification"means up to an appropriate equivalence, and for definiteness we will consider smooth (i.e. differentiable) manifolds up to diffeomorphism (equivalence by differentiable homeomorphisms). Of course, what one really seeks is an understanding of the possible phenomena, rather than a classification into long lists of cases. Simplifying things greatly we can say that

- For 2-dimensional manifolds the classification is well-known, comparatively elementary and goes back to the beginnings of the subject of topology in the 19th. century.

- The classification of "high-dimensional" manifolds, of dimension 5 or more, was to a large extent understood through the far-reaching theories developed in the middle of the 20th. century. This should not be taken too literally. In fact it is completely impractical to classify all manifolds in any useful sense: any finitely presented group can occur as the fundamental group of a manifold of dimension 4 or more so this would require something like a classification of all such groups. What we mean is that these farreaching theories translate the topological questions into algebraic ones, which are tractable in many useful cases.

- The classification of 3-dimensional manifolds might reasonably be considered to be understood through the recent resolution by Perelman of Thurston's "Geometrisation Conjecture". Again, one should qualify the degree to which this gives a precise classification. 
- For 4-dimensional manifolds a great deal is now known in the way of "examples of phenomena that can occur", but there is at present no kind of systematic picture, even at the most conjectural level.

Our focus here is on the last case above. The progress since the 1980's has largely been achieved by the introduction of new invariants, about which we say more below. These have been used to distinguish 4-manifolds which appear identical from the point of view of classical algebraic topology (homology groups, homotopy type etc.). What is completely unknown is whether these invariants are in some sense "sufficient" or, on the other hand, whether they have just moved our understanding forward by a few notches. It seems best to explain this by a concrete example, for which we take a construction of Fintushel and Stern [7] (but there are many other similar examples in the literature which one could discuss). Fintushel and Stern start with a well-known "standard" 4-manifold: a "K3 surface" $X$. Until the early 1980's it would have been reasonable to guess that any 4-manifold which has the same classical invariants as $X$ is actually diffeomorphic to $X$. But Fintushel and Stern showed that this guess is wildly out. They take any knot $K$ in ordinary 3 -space and use this to define a manifold $X_{K}$ by a certain surgery procedure (cutting out a piece of $X$ and gluing back another piece determined by $K$ ). They show that these new invariants (to be precise, the Seiberg-Witten invariants) of $X_{K}$ detect the Alexander polynomial of the knot $K$ - a well-known knot invariant dating from the 1920's and thoroughly understood in terms of the algebraic topology of the complement of $K$ in 3-space. So, first, this gives an enormous supply of mutually inequivalent 4-manifolds within the same homotopy type, since we can choose a knot to realise any prescribed Alexander polynomial. But, conversely, it is easy to find pairs $K, K^{\prime}$ of knots with the same Alexander polynomial so then we can ask whether $X_{K}$ and $X_{K^{\prime}}$ are equivalent; and this question is completely out of reach. Or, less precisely, we can ask whether there is some useful list of surgery procedures of this nature which generate all manifolds of the given homotopy type.

The outstanding problem then, in 4-manifold topology, is to find if there is something which could play the role of Thurston's Geometrisation conjecture, for the case of 3-manifolds, and which might guide further research. The key idea in Thurston's theory, and in the proof of the conjecture via "Ricci flow", is to consider structures on manifolds which are not immediately present in the topological problem. For 3-manifolds we can take these structures to be Riemannian metrics. One is obviously lead to ask: are there structures one can consider on 4-manifolds which are helpful in understanding the topology? Of course there are no very solid grounds for supposing that this is the right way forward. The royal road to understanding smooth 4-manifolds - if it exists at all-might be quite different. But in any case work in this direction leads to many other questions of great interest in their own right. Such work has followed two main paths.

One path, following most closely the 3-dimensional model, is to study the Riemannian geometry of 4-manifolds. The natural generalisation of the constant curvature metrics in 3-dimensions appearing in the Geometrisation conjecture 
are Einstein metrics in 4-dimensions, but one can also consider various other "optimal" metrics. A good deal is now known through the work of Le Brun, Anderson and others [1], [11]. Many striking special features of 4-dimensions enter through the $L^{2}$ norm of the Riemann curvature tensor (and its irreducible components). On the one hand, this is scale-invariant in four dimensions, which has important consequence for analytical questions. On the other hand, it is related via Chern-Weil theory to classical topological invariants such as the signature and Euler characteristic. A fundamental difficulty, in going from 3 dimensions to 4 , is that we do not know a classification of Einstein metrics on 4-manifolds. Thus even if, very optimistically, the Ricci flow techniques can be extended in some way to 4-dimensions-leading to, say, a decomposition of a general 4-manifold into "Einstein pieces" - this would not directly lead to purely topological conclusions. (The point is that in 3-dimensions the Ricci tensor is equivalent to the full curvature tensor, so Einstein metrics have constant curvature and the manifold is a quotient of one of three standard models, but in dimension 4 or more the curvature tensor has another component; the Weyl tensor.)

The other path is to look at symplectic structures. Indeed, the developments in 4-manifold theory since the 1980's have moved in parallel with developments in the general field of symplectic topology. In part this has to do with structural similarities between the techniques. The new 4-manifold invariants we have mentioned briefly above fall into a general pattern of enumerative invariants. They are defined by "counting" solutions to elliptic partial differential equations: the Yang-Mills instanton equation (in the earliest form of the theory) or the Seiberg-Witten equation (in most developments since 1994). In symplectic topology the analogous invariants are the Gromov-Witten invariants, defined by counting holomorphic curves, which are solutions of a variant of the CauchyRiemann equations. In both theories there are strong connections with algebraic geometry, and the long history of enumerative problems there, and with mathematical physics. Both theories bring in ideas of "Floer homology". All of this has now grown into an enormous field, in which ideas from low-dimensional topology and symplectic topology intertwine, along with much else. For example, the "Heegard" invariants of Orzsvath and Szabo, which are almost certainly equivalent to the Seiberg-Witten invariants, are based on a variant of the symplectic Floer theory construction performed within the symmetric product of a 2-manifold. Connections are emerging with another theory developed around about 1990: the Jones polynomials of knots. Khovanov discovered a knot invariant whose output is a bigraded homology group having the Jones invariant as its Euler characteristic (after collapsing one of the gradings), and Seidel and Smith [13] showed that this Khovanov homology can be, at least partially, interpreted in terms of Floer Theory. Meanwhile there are further developments, associated to contact structures on 3-manifolds, due to Eliashberg, Givental and Hofer [6] and Hutchings and Taubes. The author does not have the knowledge, nor the space here, to make any attempt to discuss all this systematically: we merely point out the general problem of understanding how all these different ideas fit together. 
Turning from the structural similarities and connections between these new techniques we can go back to ask, more directly, what can be said about the classification of symplectic structures on 4-manifolds and whether this sheds any light on the general classification problem. Again, immense advances have been made, notably through the work of Taubes [14] from the mid 1990's, but equally there are fundamental questions which we know nothing about. The simplest of Taubes' results is that (under mild technical conditions) a certain Seiberg-Witten invariant of a symplectic 4-manifold must be +1 . For example, a Fintushel-Stern manifold $X_{K}$ can only be symplectic if the Alexander polynomial has leading co-efficient 1. Further developments, combining ideas of Gromov and McDuff with Taubes' results, lead to the classification theorem of Liu [12]. We can assign an elementary numerical invariant $\kappa$ to a compact symplectic 4-manifold $(M, \omega)$

$$
\kappa=\int_{M} c_{1}(M) \wedge \omega
$$

where $c_{1}(M)$ denotes the first Chern class of the tangent bundle of $M$ (which is well-defined since the symplectic group retracts onto the unitary group). Then Liu classified symplectic manifolds with $\kappa>0$ - there are no examples beyond the standard ones furnished by rational complex surfaces and 2-sphere bundles. This is the only reasonably general classification theorem known in the subject. It is tempting to try to build on this, for example by extending to the borderline case when $\kappa=0$ (and one might expect that the only examples are the K3 surface and torus bundles), but this does not seem to be easy. One general question is whether a symplectic structure on a 4-manifold is unique, up to diffeomorphism, given the elementary topological invariants $[\omega], c_{1}(M)$

Note that these two paths, through Riemannian geometry and symplectic geometry, are not disjoint. One meeting place is in the theory of complex surfaces and Kähler geometry. In this case the invariant $\kappa$ can be thought of as the average scalar curvature, and is related to the Kodaira dimension.

\section{Kahler geometry}

A Kahler manifold is a complex manifold endowed with a Riemannian metric which is compatible with the complex structure in a natural way. First, algebraically, we require that the Riemannian inner product on each tangent space is induced by a Hermitian form. This means that the Riemannian metric can equally well be viewed as a 2 -form $\omega$, the imaginary part of the Hermitian form. Second we require a differential condition which can be expressed in various equivalent ways. One is that $\omega$ is a closed form (so defines a symplectic structure, as discussed in the previous section). Another is that the parallel transport of tangent vectors defined by the Levi-Civita connection commutes with multiplication by complex numbers (which can be phrased in terms of the holonomy group, as discussed in the next section.) In local complex co-ordinates $z_{a}$, any 
Kahler metric can be represented by a Kahler potential $\phi$;

$$
\omega=i \sum_{a b} \frac{\partial^{2} \phi}{\partial z_{a} \partial \bar{z}_{b}} d z_{a} d \bar{z}_{b} .
$$

Much work over the past half century has focused on the Ricci tensor of a Kahler manifold, which is closely related to the volume form. When the first Chern class $c_{1}(M)$ is a multiple of the Kahler class, $c_{1}=\lambda[\omega]$, one can seek a Kahler-Einstein metric with Ricci $=\lambda \omega$. Finding such a metric boils down to solving a nonlinear second order $P D E$ for the Kahler potential $\phi$. This problem was raised by Calabi in the 1950's and solved in renowned work of Yau and Aubin in the 1970's for the case when $\lambda$ is zero or negative. In the late 1980's, Tian solved the problem when $\lambda$ is positive and the complex dimension is 2 [15], but the higher dimensional case is still open.

There is another, more general, circle of questions which again goes back to work of Calabi [2]. These questions involve the existence of extremal Kähler metrics which, by definition, are the minima of the $L^{2}$ norm of the Riemann curvature tensor, viewed as a functional on the space of Kähler metrics in a fixed cohomology class $[\omega]$. Kähler-Einstein metrics are particular examples of extremal metrics, but the latter are more general, not restricted to the cases when $c_{1}=\lambda[\omega]$. The extremal condition boils down to a more formidable partial differential equation, of order 4 or 5 , depending on the precise situation and setting-up of the problem.

There are, at least, two different strands in the study of these questions. One strand emphasises Riemannian geometry. Thus a major component of Tian's analysis of Kahler-Einstein metrics on complex surfaces is a general compactness result for Einstein 4-manifolds. One has to consider Einstein metrics with

- Fixed volume;

- An upper bound on the diameter;

- An upper bound on the $L^{2}$ norm of the Riemann curvature tensor.

As we mentioned in Section 1, this third condition is particularly powerful in real dimension 4 (i.e. complex dimension 2), and less useful in higher dimensions. The first two conditions rule out local "collapsing" of the manifold, via the Bishop-Gromov inequality for the growth of the volume of geodesic balls, given a bound on the Ricci tensor. The relevant compactness theorem (proved in the late 1980's by Anderson and Nakajima) asserts that a sequence of Einstein metrics satisfying these conditions has a subsequence converging to an "orbifold" metric. Near the orbifold points the subsequence is modelled on shrinking "gravitational instantons" in a well-understood way.

The same ideas can be applied, at least to some extent, to existence questions for extremal metrics on complex surfaces. By their definition one still has control of the $L^{2}$ norm of the curvature tensor but, compared with the Einstein case above, one loses both the a priori control of the diameter and the BishopGromov theory. However, in special cases, there is another argument of Tian 
which provides an effective substitute and leads to new existence theorems [16], [4]. The general problem, going beyond these special cases, is to deal with situations where local collapsing may a priori occur. (Here "collapsing" means that the volume of a ball of small radius $r$ is much less than $r^{4}$, as occurs for example in a product $N \times S^{1}$ where $N$ is a 3-manifold and the size of the $S^{1}$ factor is small compared with $r$.) This is somewhat similar to the problem in the Ricci flow for 3-manifolds overcome by Perelman.

The second strand we discuss makes more connection with the general world of nonlinear PDE. Working in local co-ordinates, a prototype of the equations involved in the Kahler-Einstein theory is the complex Monge-Ampère equation

$$
\operatorname{det}\left(\frac{\partial^{2} \phi}{\partial z_{a} \partial \bar{z}_{b}}\right)=1
$$

Similarly, it turns out that a prototype for the extremal equation is a 4th. order PDE for the Kahler potential $\phi$ which can be neatly written as the system

$$
\sum \phi^{a b} \frac{\partial^{2} L}{\partial z_{a} \partial \bar{z}_{b}}=0 \quad ; \quad L=\log \operatorname{det}\left(\frac{\partial^{2} \phi}{\partial z_{a} \partial \bar{z}_{b}}\right) .
$$

(This system defines metrics of zero scalar curvature, which are prototypes of extremal metrics. We use the notation $\phi^{a b}$ for the inverse of the matrix $\frac{\partial^{2} \phi}{\partial z_{a} \partial \bar{z}_{b}}$.)

There is a well-known analogy in which one replaces the function $\phi$ on domain in $\mathbf{C}^{n}$ by a function $f$ on a domain in $\mathbf{R}^{n}$ and the differential operator $\frac{\partial^{2}}{\partial z_{a} \partial \bar{z}_{b}}$ by the ordinary Hessian $\frac{\partial^{2}}{\partial x_{i} \partial x_{j}}$. Thus the analogue of (1) is, of course, the real Monge-Ampère equation

$$
\operatorname{det}\left(\frac{\partial^{2} f}{\partial x_{i} \partial x_{j}}\right)=1
$$

for a convex function $f$. The second equation (2) can be viewed as a special case of a family of 4th. order PDE's; with both real and complex versions. We take any convex function $S(t)$ of one real variable and consider (formally) the functionals

$$
\mathcal{F}_{\mathbf{R}}=\int_{\mathbf{R}^{n}} S\left(\operatorname{det}\left(\frac{\partial^{2} f}{\partial x_{i} \partial x_{j}}\right)\right) \quad, \quad \mathcal{F}_{\mathbf{C}}=\int_{\mathbf{C}^{n}} S\left(\operatorname{det}\left(\frac{\partial^{2} \phi}{\partial z_{a} \partial \bar{z}_{b}}\right)\right)
$$

in the two cases respectively. The Euler-Lagrange equations $\delta \mathcal{F}_{\mathbf{R}}=0, \delta \mathcal{F}_{\mathbf{C}}=0$ are equations which can be written in the same shape as (2), or the obvious real analogue. The equation (2) occurs, in the complex case, when $S(t)=t \log t$. Another example studied in the literature, in the real case, is the equation defining an "affine maximal" hypersurface in $\mathbf{R}^{n+1}$. Other equations of this type have been studied by Trudinger and Wang [17].

There is an enormous body of theory dealing with real Monge-Ampère equations. One celebrated result, proved by Jörgens in dimension 2 and by Calabi and Pogorelov in higher dimensions, is that the only solutions of (3) on the whole 
of $\mathbf{R}^{n}$ are the obvious ones: quadratic functions. A complex analogue of this, for solutions of (1) on $\mathbf{C}^{n}$, would be an important step towards understanding Kahler-Einstein metrics on compact manifolds. (The passage from a compact manifold to $\mathbf{C}^{n}$ occurs when one makes a rescaling or "blow-up" argument.) But this complex analogue is not known.

To sum up, from the PDE point of view, these questions in Kähler geometry lead one, first, to try to extend the deeper parts of the real Monge-Ampère theory to the complex case and, second, to study the more formidable 4 th. order equations like (2).

\section{Special holonomy and calibrated geometry}

The Levi-Civita connection of a Riemannian manifold $M$ defines the operation of parallel transport of tangent vectors along paths. Parallel transport around a loop starting and ending at some fixed base point $p$ gives an orthogonal transformation of the tangent space $T M_{p}$ and the set of all such transformations is a Lie subgroup of the orthogonal group: the holonomy group of the Riemannian manifold. We can easily reduce to a case when this group is connected. In the 1950 's Berger discovered that there is only a short list of possible holonomy groups (leaving aside the rigid "symmetric spaces"). The generic case, when $M$ has dimension $n$, is the full group $S O(n)$. If $n=2 m$ is even and $M$ is a Kähler manifold of complex dimension $m$ one gets, generically, holonomy $U(m)$ while if the Ricci tensor vanishes one gets $S U(m)$. If $n$ is divisible by 4 there are two other families, involving quaternionic structures. Beyond these 5 families there are just two exceptional cases, in dimensions 7 and 8 . These are connected to special algebraic structures involving the Cayley numbers but they also fit into a pattern of low-dimensional phenomena related to spinors. In each dimension $n$ we have a spin representation $S$ of $\operatorname{Spin}(n)$ (the double cover of $S O(n)$ ) which comes with various algebraic structures depending on the reduction of $n$ modulo 8. For $n \leq 6$ the spin representation is so small that this leads to exceptional isomorphisms with other familiar Lie groups

$\operatorname{Spin}(3)=S U(2), \operatorname{Spin}(4)=S U(2) \times S U(2), \operatorname{Spin}(5)=S p(2), \operatorname{Spin}(6)=S U(3)$.

When $n=7$ the spin representation gives a subgroup $\operatorname{Spin}(7) \subset S O(8)$ and this is the exceptional holonomy group in 8 dimensions featuring on Berger's list. This subgroup $\operatorname{Spin}(7)$ acts transitively on the unit sphere in $\mathbf{R}^{8}$ and the stabiliser of a point defines a subgroup $G_{2} \subset \operatorname{Spin}(7)$ which, it turns out, can be viewed as a subgroup $G_{2} \subset S O(7)$. This is the other exceptional holonomy group, in 7 dimensions. The study of Riemannian metrics with these exceptional holonomy groups $G_{2}$, $\operatorname{Spin}(7)$ is a very active field, with intriguing special phenomena which, as outlined above, can perhaps be seen as lying on the borderline between "low dimensional" and "high dimensional" Riemannian geometry; in a similar vein to our topological discussion in Section 1. 
From now on we restrict attention to the case of 7 -manifolds and holonomy $G_{2}$. The definition sketched above means that one characterisation of these is as manifolds with a parallel spinor field, but there are many other characterisations which turn out to be equivalent. From one point of view, one can regard the structure as being a "cross-product" on tangent vectors, modelled on the imaginary Cayley numbers and analogous to the familiar cross product on $\mathbf{R}^{3}$. From another point of view one can regard the structure as being a distinguished 3-form $\phi$ on $M$. This determines, by a purely algebraic procedure, a Riemannian metric $g(\phi)$ and hence a 4 -form $*_{g(\phi)} \phi$. The differential condition, analogous to that in Kahler geometry, is that $\phi$ and $*_{g(\phi)} \phi$ are both closed.

The global theory of these Riemannian manifolds really began with work of Joyce [8], who constructed compact examples. A different construction, producing further examples, was found by Kovalev [10]. Their work brings to the fore the obvious question of classifying all compact manifolds with holonomy $G_{2}$. More precisely, one would like to know

- Which compact 7-manifolds admit a metric with holonomy $G_{2}$ ?

- If $M$ is such a manifold, describe the "moduli space" $\mathcal{M}$ of all such metrics, modulo diffeomorphism. (To be more precise, we will take diffeomorphisms isotopic to the identity.)

In connection with the first item, various constraints on the topology are known: in particular the main questions involve simply connected manifolds. Thus, as outlined in Section 1, we have quite a good hold on the topological classification of the 7-manifolds in questions, especially when $H_{2}(M)=0$. In connection with the second item, we have a map $\pi: \mathcal{M} \rightarrow H^{3}(M ; \mathbf{R})$ taking the de Rham cohomology class of $\phi$ (somewhat analogous to the Kahler class $[\omega]$ in Section 2 ) and it is known that this is a local diffeomorphism. Thus the image of $\pi$ is an open set $U \subset H^{3}(M)$. A refinement of the second question is to ask for a description of $U$ and whether in fact $\pi$ is a global diffeomorphism from $\mathcal{M}$ to $U$.

Little is known about these questions. Compactness results for Riemannian metrics, similar to those discussed in Section 2, are very relevant to the second question, and much progress in this direction has been made by Cheeger and Tian [3]. Suppose we have a sequence of manifolds of holonomy $G_{2}$, normalised to volume 1, with bounded diameter. Then Cheeger and Tian show that there is a subsequence which converges to a possibly singular space with singularities of codimension at least 4 . Roughly speaking, the picture transverse to the singular set, at a generic point, is the same as that encountered for Einstein 4-manifolds, modelled on shrinking gravitational instantons. Thus, if we have a sequence in $\mathcal{M}$ and take a suitable subsequence, either we see this kind of "orbifold convergence" or the diameter must tend to infinity. This alternative fits in well with the known examples. The constructions of Joyce and Kovalev are by analytical "gluing" methods, which essentially analyse a neighbourhood of a point on the boundary of the set $U$. Joyce's construction yield explicit sequences of the first kind, and Kovalev's of the second. 
These exceptional structures in dimensions 7 and 8 fit into another general picture of "calibrated geometry" due to Harvey and Lawson. What this means is that one can define special classes of minimal submanifolds, satisfying systems of first order PDE. The prototype is the class of complex submanifolds of a Kahler manifold, which are always minimal. Another class is furnished by "special Lagrangian" submanifolds in an ambient manifold of holonomy $S U(m)$. In a manifold of holonomy $G_{2}$ we have "associative" 3-dimensional submanifolds and "co-associative" 4-dimensional submanifolds. There are similar higher-dimensional analogous of the Yang-Mills instanton equations [5]. These theories have the basic differential-geometric properties required to define "enumerative invariants" of the kind discussed in Section 1. These should, roughly speaking, "count" special Lagrangian submanifolds, co-associative submanifolds etc. A theory of this kind could be expected to have applications to the classification problems for manifolds with special holonomy, and would fit in with developments in the theoretical physicists M-theory. A great deal of work has been done in this direction, notably by Joyce on special Lagrangian submanifolds [9], but the outstanding overall difficulty involves compactness questions, of a similar general nature to those for Riemannian metrics discussed above. It is clear that, a priori one has to consider singular solutions but there are serious obstacles in extending the differential-geometric constructions to these. Going back to the prototype example of complex submanifolds: we are familiar with the notion of a singular complex subvariety and we can call upon all the resources of commutative algebra and algebraic geometry to handle them. In these other calibrated geometries we expect to encounter phenomena of equal complexity, but we do not have recourse to the same algebraic machinery.

\section{References}

[1] Anderson, M.T. Canonical metrics on 3-manifolds and 4-manifolds Asian Jour. Math. 102006 127-163

[2] Calabi, E. Extremal Kahler metrics In: Seminar in Differential Geometry (Ed. S-T.Yau) Annals of Math. Studies Princeton UP 1982

[3] Cheeger, J. and Tian, G. Anti-self-duality of curvature and degeneration of metrics with special holonomy Commun. Math. Phys. 2552005 391-417

[4] Chen, X., LeBrun, C. and Weber, B. On conformally Kahler, Einstein, manifolds Arxiv DG/ 07050710

[5] Donaldson, S. and Thomas, R. Gauge theory in higher dimensions In: The Geometric Universe: Science, Geometry and the work of Roger Penrose. Huggett et al eds. Oxford UP 1998 31-47 
[6] Eliashberg, Y., Givental, A. and Hofer, H. Introduction to symplectic field theory Geometric and Functional Analysis, Special issue, vol. 22000 560672

[7] Fintushel, R, and Stern, R. Knots, links and four-manifolds Inventiones Math. 1341998 363-400

[8] Joyce, D. Compact manifolds with special holonomy Oxford U.P. 2000

[9] Joyce, D. Special Lagrangian singularities In: Different faces of geometry Kluwer 2004 163-198

[10] Kovalev, A. Twisted connected sums and special Riemannian holonomy J. Reine Angew. Math. 5652003 125-160

[11] LeBrun, C. Curvature functionals, optimal metrics, and the differential topology of 4-manifolds In: Different faces of geometry Kluwer 2004 199256

[12] Liu, A-K. Some new applications of general wall-crossing formula, Gompf's conjecture and applications Math. Res. Letters 3 (1996) 569-85

[13] Seidel, P and Smith, I A link invariant from the symplectic geometry of nilpotent slices Duke Math. Jour. 1342006 453-514

[14] Taubes, C.H. Seiberg-Witten and Gromov invriants for symplectic fourmanifolds International Press 2000

[15] Tian, G. On Calabi's conjecture for complex surfaces with positive first Chern class Inventiones Math. 1011990 101-172

[16] Tian, G. and Viaclovsky, J. Moduli spaces of critical Riemannian metrics in dimension four Advances in Math. 1962005 346-372

[17] Trudinger, N. S. and Wang, X-J. Bernstein-Jörgens theorem for a fourth order partial differential equation J. Partial Differential Equations 152002 $78-88$ 